\title{
Prognostic Implications of QRS Duration in Third-Degree Atrioventricular Block Patients with Heart Failure Treated with Cardiac Resynchronization Therapy
}

\author{
Kangyu Chen, ${ }^{1} \mathrm{MD}$, Hao Su, ${ }^{1} \mathrm{MD}$, Cuiping Xie, ${ }^{1} \mathrm{MD}$, Qi Wang, ${ }^{1} \mathrm{MD}$, Fei Yu, ${ }^{1} \mathrm{MD}$, Qi Sun, ${ }^{1} \mathrm{MD}$, \\ Hongjun Zhu, ${ }^{1} \mathrm{MD}$, Dongmei Yang, ${ }^{2} \mathrm{MD}$, Jian $\mathrm{Xu},{ }^{1} \mathrm{MD}$ and Ji Yan, ${ }^{1} \mathrm{MD}$
}

\begin{abstract}
Summary
Cardiac resynchronization therapy (CRT) improves heart function and prognosis in third-degree atrioventricular block (AVB) patients with heart failure (HF). However, it is still unclear how to screen for appropriate patients before implantation. This study aimed to evaluate the value of using QRS duration to predict CRT efficacy.

This study enrolled a total of 72 third-degree AVB patients with HF who received CRT implantation. The patients were divided into Groups A (QRS duration $<120 \mathrm{~ms}, 33$ cases), B (120 ms $\leq$ QRS duration $<150 \mathrm{~ms}$, 22 cases), and $\mathrm{C}$ (QRS duration $\geq 150 \mathrm{~ms}, 17$ cases) according to their baseline QRS duration. The effects of different QRS durations on CRT efficacy were analyzed.

The CRT response rate were $30.3 \%, 50.0 \%$, and $76.5 \%$ in Groups A, B, and C, respectively $(P=0.008)$. The patients in the 3 groups showed significant changes in left ventricular (LV) end-diastolic volume, LV endsystolic volume, and LV ejection fraction over the baseline values at 12 months after the implantation $(P<$ 0.05 ), with the greatest change observed in Group C. Survival analysis indicated statistically significant differences among Groups $\mathrm{A}, \mathrm{B}$, and $\mathrm{C}(P=0.024)$. Multivariate logistic regression analysis suggested that $\mathrm{QRS}$ duration was an independent prognostic factor for CRT efficacy. Baseline QRS duration was associated with improved myocardial remodeling and reductions in the incidence rates of primary endpoint events.

QRS $\geq 150 \mathrm{~ms}$ is an effective predictor of postoperative outcome in patients with third-degree AVB and HF treated with CRT.
\end{abstract}

Key words: Electrical dyssynchrony, Reverse remodeling, Non-response

(Int Heart J 2018; 59: 1320-1326)

$\mathrm{P}$ ermanent pacing is an effective treatment for thirddegree atrioventricular block (AVB). However, the traditional right ventricular apex pacing may cause artificial dyssynchrony, which further leads to myocardial remodeling and cardiac enlargement and gradually develops to clinical heart failure (HF), especially in patients with decreased basic heart functions. ${ }^{1.2}$ Right ventricular septal pacing can theoretically reduce the risk of cardiac dyssynchrony, but its efficacy is still controversial due to positioning accuracy, among other reasons. ${ }^{3.4)}$

Cardiac resynchronization therapy (CRT) improves cardiac synchronization in chronic HF patients complicated with dyssynchrony and reduces hospitalization rates and all-cause mortality. For patients with third-degree or greater AVB accompanied by decreased cardiac function, direct implantation of a CRT device can delay or even reverse myocardial remodeling and improve prognosis. ${ }^{5-8)}$
However, it is still unclear which patients benefit from CRT in this patient population. Identifying reliable predictors for preoperative screening of patients will be conducive to decreasing operative risk and medical costs. QRS duration is one of the core indicators for assessment of cardiac systolic dyssynchrony. This study aimed to evaluate the value of QRS duration in predicting the efficacy of direct implantation of CRT in patients with third-degree AVB accompanied by HF.

\section{Methods}

Enrolled subjects: We analyzed prospectively collected data from patients who received CRT implantation at the Anhui Provincial Hospital, China, between January 2010 and May 2016 due to third-degree AVB accompanied by HF. The inclusion criteria were 1) third-degree AVB; 2)

From the ${ }^{1}$ Department of Cardiology, The Provincial Hospital Affiliated to Anhui Medical University, Hefei, China and ${ }^{2}$ Echocardiography Laboratory, The Provincial Hospital Affiliated to Anhui Medical University, Hefei, China.

This study was supported by the Central Guidance for Local Science and Technology Development Program Special Funds (2016080802D113) and the Anhui Province Key Science and Technology Research Project (1301042210).

Address for correspondence: Ji Yan, MD, Department of Cardiology, The Provincial Hospital Affiliated to Anhui Medical University, No. 17, Lujiang Road, Hefei, Anhui, China. E-mail: yanji111111@126.com

Received for publication October 10, 2017. Revised and accepted February 16, 2018.

Released in advance online on J-STAGE October 25, 2018.

doi: 10.1536/ihj.17-577

All rights reserved by the International Heart Journal Association. 
left ventricular $(\mathrm{LV})$ ejection fraction $(\mathrm{LVEF})<50 \%$ and LV end-diastolic diameter (LVEDD) $>55 \mathrm{~mm}$. The patients who had acute coronary syndromes, coronary revascularization within 3 months prior to the study, myocarditis within 6 months prior to the study, or severe liver or renal failure (serum creatinine $>265.2 \mu \mathrm{mol} / \mathrm{L}$ ) were excluded from this study. This study was approved by the Ethics Committee of the Anhui Provincial Hospital, and all enrolled patients signed the informed consent.

Clinical assessment: The baseline data were collected from all patients and recorded. A standard 12-lead electrocardiogram was examined under the resting state, with a paper speed at $50 \mathrm{~mm} / \mathrm{s}$ and an amplitude scale at $10 \mathrm{~mm} /$ $\mathrm{mV}$. The heart rhythm and heart rate were recorded. The maximum value of QRS duration of the 12 leads was considered as the QRS duration to be analyzed. ${ }^{9)}$ The parameters were measured by 2 cardiologists and inconsistent data were resolved through discussion between them. The patients were divided into Groups A (QRS duration $<120$ $\mathrm{ms})$, B $(120 \mathrm{~ms} \leq \mathrm{QRS}$ duration $<150 \mathrm{~ms})$, and $\mathrm{C}$ (QRS duration $\geq 150 \mathrm{~ms}$ ) according to their QRS durations. Using a Philips iE33 Ultrasound System, the LVEDD and LV end-systolic diameter (LVESD) were measured in the $\mathrm{M}$ mode, the pulmonary artery systolic pressure was estimated through tricuspid regurgitation rate, and the LV end-diastolic volume (LVEDV), LV end-systolic volume (LVESV) and LVEF were measured in 3-dimensional mode.

CRT implantation and optimization: The CRT device (St. Jude Medical, Saint Paul, MN, USA) was implanted via an intravenous route in all patients. The procedure was started with a subclavian venous puncture or cephalic venous incision, followed by retrograde coronary angiography to fully display all coronary venous branches. The LV lead was placed in the lateral vein, the posterolateral vein, or their adjacent branch, and was confirmed to have no phrenic nerve stimulation at $10 \mathrm{~V}$. The right ventricular lead was implanted in the right ventricle apex or septal wall, while the right atrial lead was placed at the right atrial appendage. All of the leads were fixed after satisfactory parameters were obtained from the tests. The leads were then connected to the pulse generator, the skin was sutured layer by layer, and the surgical site was dressed with compression. The postoperative sensed and paced atrioventricular delays were set to 100 and $130 \mathrm{~ms}$, respectively. The optimal interventricular delay was defined as the narrowest interval of the QRS complex in biventricular pacing.

Follow-up visits: The postoperative follow-up visits at the pacemaker clinic were performed regularly at $1,3,6$, and 12 months after implantation. Follow-up visits for discomfort were added when the patients experienced discomfort. At the follow-up visits, the leads and pacing-related parameters were controlled by programs to ensure effective biventricular pacing. Echocardiography was repeated at the follow-up 12 months after surgery, and the examination method and measured parameters were the same as described above. The primary endpoints of the study were emergency or hospitalization requiring intravenous medication due to HF and all-cause death. Effective CRT was defined as a reduction of $\geq 15 \%$ in the LVESV at 12 months after surgery. ${ }^{7,10}$

Statistical analysis: Data processing and statistical analysis were performed using the software SPSS 17.0. Count data were expressed as rates or percentages, and their comparison was performed using the chi-squared test. Measurement data of normal distribution were expressed as means \pm standard deviations; comparisons between 2 groups were performed using the paired $t$-test, while comparisons of multiple groups were performed using analysis of variance. Measurement data of non-normal distribution were expressed as medians $\left(\mathrm{P}_{25}, \mathrm{P}_{75}\right)$, and comparisons of multiple groups were performed using the Mann-Whitney $U$ test. Statistical analysis of influencing factors was performed using univariate and multivariate logistic regression analyses. The assessment of survival was performed using the log-rank test of the Kaplan-Meier survival analysis. Differences of $P<0.05$ were considered statistically significant.

\section{Results}

Baseline information: A total of 77 patients were enrolled in the study. Five patients were excluded due to incomplete data, and 72 patients were eventually included in the analyses, with 33 patients in Group A, 22 patients in Group B, and 17 patients in Group C. The QRS durations of Groups A, B, and C were 100.4 \pm 9.4, $133.6 \pm 7.7$, and $162.1 \pm 9.6 \mathrm{~ms}$, respectively. The baseline data of the patients are shown in Table I. No significant differences were found in sex, age, New York Heart Association (NYHA) classification, heart rate, atrial fibrillation and complications among the patients in the 3 groups (Table I). No statistically significant differences were observed in the baseline echocardiography parameters among the 3 groups (Table II).

Analysis of QRS duration: The overall QRS duration of the patients increased from $121.5 \pm 26.7$ to $142.6 \pm 10.8$ ms $(P<0.001)$ following biventricular pacing, and the QRS durations after CRT implantation were significantly different from the baseline in all 3 groups $(P<0.01$ in all groups). Differences were observed in the changes of postoperative QRS duration over baseline among the 3 groups, with statistical significance $(P<0.001)$. QRS widening was the most obvious in Group A, while QRS duration was significantly shortened in Group C (Table III).

CRT efficacy: At the 12-month follow-up after the implantation, the overall LVEDV, LVESV, LVEDD, LVESD, LVEF, and systolic pulmonary artery pressure (SPAP) were all improved in the patients, with no significant differences between the 3 groups. Compared with the values before the implantation, LVEDV, LVESV, LVEDD, LVESD, LVEF, and SPAP were only improved in Group $\mathrm{C}$ (Table II). The differences before and after the implantation were compared between the 3 groups (Figure 1), and Group $\mathrm{C}$ exhibited greater improvements in the above parameters than the other 2 groups $(P<0.05)$. The percentage of NYHA I, II, and III was $58.6 \%, 31.4 \%$, and $10.0 \%$, respectively after CRT. In comparison to the preimplantation values, CRT significantly improved NYHA functional class $(P<0.01)$, but there were no significant 
Table I. Baseline Characteristics of the Study Patients with Different QRS Durations

\begin{tabular}{|c|c|c|c|c|c|}
\hline & $\begin{array}{l}\text { Total } \\
(n=72)\end{array}$ & $\begin{array}{c}\mathrm{A}(n=33) \\
(\mathrm{QRS}<120 \mathrm{~ms})\end{array}$ & $\begin{array}{c}\mathrm{B}(n=22) \\
(120 \mathrm{~ms} \leq \mathrm{QRS}<150 \mathrm{~ms})\end{array}$ & $\begin{array}{c}\mathrm{C}(n=17) \\
(\mathrm{QRS} \geq 150 \mathrm{~ms})\end{array}$ & $P$ value \\
\hline Male, $n(\%)$ & $40(55.6)$ & 19 & 11 & 10 & 0.817 \\
\hline Age, years & $62.9 \pm 13.1$ & $62.6 \pm 14.4$ & $62.8 \pm 13.2$ & $63.5 \pm 10.5$ & 0.976 \\
\hline NYHA class, $n(\%)$ & & & & & 0.995 \\
\hline I & $15(20.8)$ & $6(18.2)$ & $5(22.7)$ & $3(17.6)$ & \\
\hline II & $43(59.7)$ & $20(60.6)$ & $12(54.5)$ & $10(58.8)$ & \\
\hline III & $14(19.4)$ & $7(21.2)$ & $5(22.7)$ & $4(23.5)$ & \\
\hline Heart rate (beats/minute) & $43.1 \pm 5.1$ & $43.9 \pm 6.0$ & $41.6 \pm 4.2$ & $43.5 \pm 4.1$ & 0.277 \\
\hline $\mathrm{AF}, n(\%)$ & $10(13.9)$ & $5(15.2)$ & $2(9.1)$ & $3(17.6)$ & 0.751 \\
\hline CHD, $n(\%)$ & $10(13.9)$ & $6(18.2)$ & $2(9.1)$ & $2(11.8)$ & 0.682 \\
\hline Hypertension, $n(\%)$ & $32(44.4)$ & $14(43.8)$ & $11(50.0)$ & $7(41.2)$ & 0.817 \\
\hline DCM, $n(\%)$ & $18(25.0)$ & $7(21.2)$ & $7(31.8)$ & $4(23.5)$ & 0.259 \\
\hline Diabetes, $n(\%)$ & $16(22.2)$ & $10(30.3)$ & $3(13.6)$ & $3(17.6)$ & 0.116 \\
\hline $\mathrm{SCr}, \mu \mathrm{mol} / \mathrm{L}$ & $84.1 \pm 30.0$ & $83.7 \pm 33.1$ & $84.7 \pm 17.2$ & $83.8 \pm 37.6$ & 0.994 \\
\hline
\end{tabular}

Table II. Baseline and 12-Month Follow-up Echocardiographic Parameters of the Study Patients with Different QRS Durations

\begin{tabular}{lccccc}
\hline & $\begin{array}{c}\text { Total } \\
(n=72)\end{array}$ & $\begin{array}{c}\mathrm{A}(n=33) \\
(\mathrm{QRS}<120 \mathrm{~ms})\end{array}$ & $\begin{array}{c}\mathrm{B}(n=22) \\
(120 \mathrm{~ms} \leq \mathrm{QRS}<150 \mathrm{~ms})\end{array}$ & $\begin{array}{c}\mathrm{C}(n=17) \\
(\mathrm{QRS} \geq 150 \mathrm{~ms})\end{array}$ & $P$ value \\
\hline LVEDV & & & & & \\
$\quad$ Baseline & $222.5 \pm 51.5$ & $213.8 \pm 49.6$ & $220.6 \pm 34.3$ & $241.8 \pm 69.0$ & 0.187 \\
$\quad$ Follow-up & $209.0 \pm 64.3^{*}$ & $218.1 \pm 72.5$ & $204.1 \pm 58.4$ & $197.8 \pm 55.2^{\#}$ & 0.529 \\
LVESV & & & & & \\
$\quad$ Baseline & $137.2 \pm 39.1$ & $128.5 \pm 35.8$ & $138.9 \pm 35.5$ & $151.9 \pm 46.8$ & 0.131 \\
$\quad$ Follow-up & $126.5 \pm 55.5^{*}$ & $135.2 \pm 59.8^{\#}$ & $124.5 \pm 53.4$ & $112.4 \pm 48.9^{\#}$ & 0.383 \\
LVEDD & & & & & \\
Baseline & $65.4 \pm 6.4$ & $64.3 \pm 6.2$ & $65.1 \pm 4.9$ & $67.8 \pm 8.1$ & 0.188 \\
Follow-up & $63.7 \pm 8.7^{*}$ & $65.0 \pm 9.1$ & $62.4 \pm 8.2$ & $62.6 \pm 8.8^{\#}$ & 0.474 \\
LVESD & & & & & \\
Baseline & $52.9 \pm 6.7$ & $51.6 \pm 6.2$ & $52.8 \pm 6.0$ & $55.4 \pm 8.0$ & 0.171 \\
Follow-up & $51.1 \pm 8.9^{*}$ & $52.0 \pm 9.7$ & $50.9 \pm 8.7$ & $49.6 \pm 7.8^{\#}$ & 0.657 \\
LVEF & & & & & \\
Baseline & $38.6 \pm 7.0$ & $40.1 \pm 6.3$ & $37.5 \pm 7.3$ & $37.1 \pm 7.5$ & 0.249 \\
Follow-up & $41.2 \pm 10.1^{\#}$ & $39.7 \pm 8.9$ & $40.8 \pm 10.1$ & $44.4 \pm 12.0^{\#}$ & 0.295 \\
PSAP & & & & & \\
$\quad$ Baseline & $42.6 \pm 9.6$ & $42.3 \pm 10.9$ & $43.1 \pm 9.1$ & $42.5 \pm 7.9$ & 0.954 \\
Follow-up & $40.8 \pm 10.5^{\#}$ & $41.7 \pm 11.4$ & $41.7 \pm 10.4$ & $38.1 \pm 8.5^{\#}$ & 0.459 \\
\hline
\end{tabular}

Table III. Changes of Postoperative QRS Duration over Baseline among the 3 Groups

\begin{tabular}{lccccr}
\hline & $\begin{array}{c}\text { Total } \\
(n=72)\end{array}$ & $\begin{array}{c}\mathrm{A}(n=33) \\
(\mathrm{QRS}<120 \mathrm{~ms})\end{array}$ & $\begin{array}{c}\mathrm{B}(n=22) \\
(120 \mathrm{~ms} \leq \mathrm{QRS}<150 \mathrm{~ms})\end{array}$ & $\begin{array}{c}\mathrm{C}(n=17) \\
(\mathrm{QRS} \geq 150 \mathrm{~ms})\end{array}$ & $P$ value \\
\hline Baseline QRS, ms & $125.1 \pm 26.7$ & $100.4 \pm 9.4$ & $133.6 \pm 7.6$ & $162.1 \pm 9.6$ & $<0.001$ \\
Paced QRS, ms & $142.6 \pm 10.8^{\#}$ & $138.9 \pm 7.0^{\#}$ & $142.2 \pm 12.6^{*}$ & $150.1 \pm 10.9^{\#}$ & 0.002 \\
$\Delta$ QRS, ms & $25(-4.0,36.0)$ & $38.0(33.0,45.0)$ & $11.0(-9.0,24.5)$ & $-18.0(-27.0,7.0)$ & $<0.001$ \\
\hline
\end{tabular}

Compared to baseline, $* P<0.05,{ }^{\sharp} P<0.01$.

differences between the 3 groups.

According to the definition of effective CRT, the treatment was effective in $34(47.2 \%)$ patients, and the response rates of Groups $\mathrm{A}, \mathrm{B}$, and $\mathrm{C}$ were $30.3 \%, 50.0 \%$, and $76.5 \%$, respectively, with statistically significant differences $(P=0.008)$. Group $C$ showed better CRT efficacy than the other 2 groups. The results of univariate analysis showed that QRS duration, sex, LVEF, and SPAP were possible factors influencing the efficacy of CRT $(P<$ $0.10)$. When these factors were introduced into a multi- variate logistic regression model, QRS duration, sex, and SPAP were eventually indicated as the factors that affected CRT efficacy (Table IV).

During the 12-month follow-up, a total of 28 $(38.9 \%)$ patients reached the endpoint of follow-up, with 26 cases of emergency or hospitalization that required intravenous medication due to $\mathrm{HF}$ and 2 cases of death. In total, 18, 7, and 3 patients reached the primary endpoint in Groups A, B, and C, respectively. As shown in Figure 2 , the survival analysis indicated a statistically significant 
LVEDV

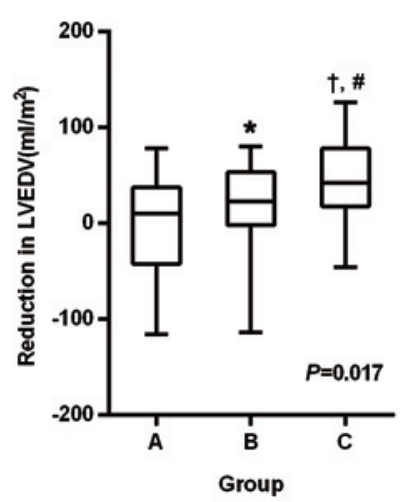

LVESD

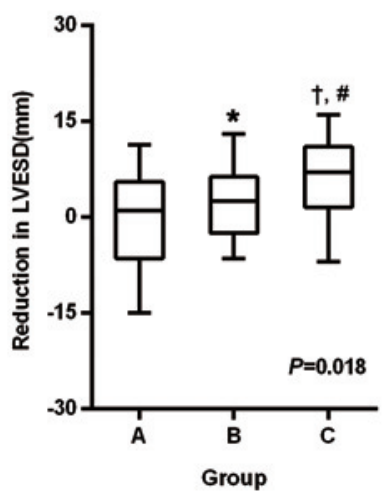

LVESV

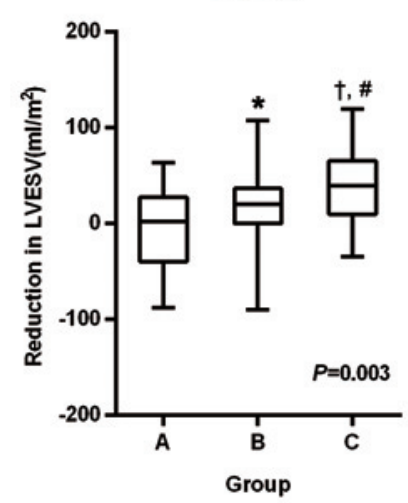

LVEF

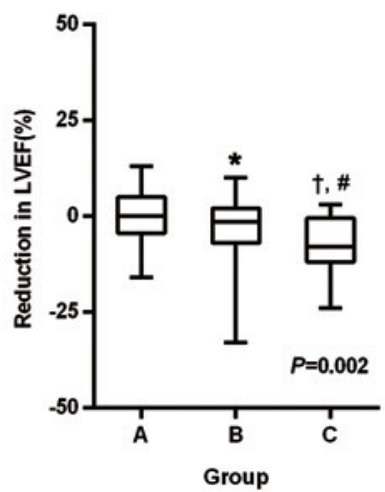

LVEDD
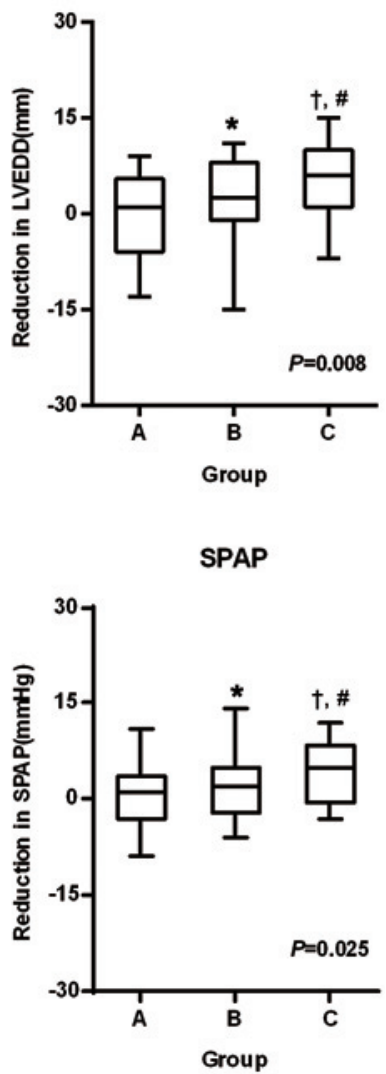

Figure 1. Changes in echocardiographic parameters after CRT. Groups A (QRS duration $<120 \mathrm{~ms}), \mathrm{B}(120 \mathrm{~ms} \leq$ QRS duration $<150 \mathrm{~ms}$ ), and $\mathrm{C}$ (QRS duration $\geq 150 \mathrm{~ms}$ ); ${ }^{*} P>0.05$ compared to group $\mathrm{A},{ }^{\dagger} P>0.05$ compared to group $\mathrm{B},{ }^{\#} P<0.05$ compared to group $\mathrm{A}$.

Table IV. Univariate and Multivariate Logistic Regression Analyses for the Response to Cardiac Resynchronization Therapy

\begin{tabular}{|c|c|c|c|c|}
\hline & \multicolumn{2}{|l|}{ Univariate } & \multicolumn{2}{|c|}{ Multivariate } \\
\hline & HR $(95 \%$ CI $)$ & $P$ value & HR $(95 \%$ CI $)$ & $P$ value \\
\hline Sex & $0.303(0.114-0.807)$ & 0.017 & $0.273(0.087-0.857)$ & 0.026 \\
\hline Age & $0.972(0.937-1.009)$ & 0.137 & & \\
\hline $\mathrm{AF}$ & $0.429(0.101-1.811)$ & 0.249 & & \\
\hline CHD & $1.138(0.299-4.329)$ & 0.850 & & \\
\hline QRS group & $1.964(1.069-3.607)$ & 0.030 & $2.679(1.284-5.601)$ & 0.009 \\
\hline LVEDV & $1.001(0.992-1.010)$ & 0.836 & & \\
\hline LVESV & $0.996(0.986-1.008)$ & 0.499 & & \\
\hline LVEF, \% & $1.071(0.997-1.150)$ & 0.059 & $1.070(0.980-1.168)$ & 0.130 \\
\hline SPAP, $\mathrm{mmHg}$ & $0.937(0.888-0.990)$ & 0.021 & $0.930(0.872-0.991)$ & 0.026 \\
\hline
\end{tabular}

difference between the 3 groups $(P=0.024)$.

\section{Discussion}

The subjects included in this study were patients with third-degree AVB accompanied by HF. The results of the study showed that: (1) implantation of CRT in these patients significantly improved cardiac functions, but with variations in efficacy, and some patients exhibited poor ef- ficacy; (2) baseline QRS duration was an independent predictor of CRT efficacy; and (3) patients with QRS duration $\geq 150 \mathrm{~ms}$ showed better CRT response rates, reversal of myocardial remodeling, and prognosis than patients with QRS duration $<150 \mathrm{~ms}$.

Cardiac pacing is an effective treatment for patients with bradyarrhythmia, but pacing-induced cardiomyopathy after right ventricular pacing continues to be reported in the literature, especially in pacing-dependent patients with 


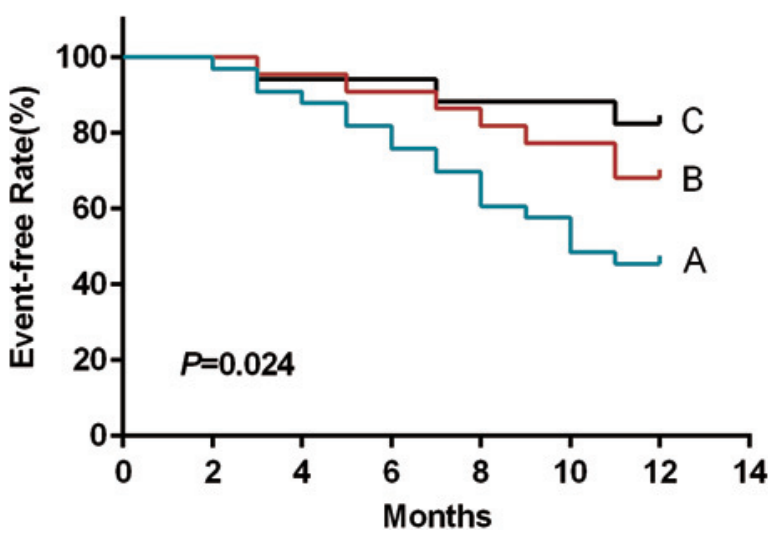

Figure 2. Freedom from a Primary-Outcome Event. Groups A (QRS duration $<120 \mathrm{~ms})$, B $(120 \mathrm{~ms} \leq$ QRS duration $<150 \mathrm{~ms})$, and C (QRS duration $\geq 150 \mathrm{~ms}$ ).

decreased cardiac functions. ${ }^{11-14)}$ In recent years, selective site pacing from the right ventricular mid-septum has made rapid progress, and some studies suggest that it results in better cardiac function and prognosis than the right ventricular apical pacing. ${ }^{15,16)}$ However, based on the existing information from evidence-based medicine, right ventricular septal pacing did not show significant advantages. ${ }^{417)}$ The CRT that has gradually been applied to clinical use after 2000 has been shown to significantly improve the cardiac functions and prognosis of the HF patients with cardiac dyssynchrony. CRT implantation in patients with AVB accompanied by heart failure has been reported in the literature since $2005 .^{5,18)}$ The publication of the results related to the BLOCK-HF study ${ }^{6,7,19)}$ confirmed that CRT could significantly improve the clinical symptoms, myocardial remodeling, and prognosis of patients with AVB accompanied by heart failure. Consistent with the above findings, the results of this study demonstrated that CRT significantly improved the myocardial remodeling and prognosis of the patients with third-degree AVB accompanied by HF.

However, in clinical practice, CRT does not show desirable efficacy in all patients with AVB accompanied by HF. The BLOCK-HF study ${ }^{7}$ assessed the efficacy of CRT in patients with AVB accompanied by heart failure using the LVESV index (LVESVI) and found that only $43 \%$ of the patients had reductions of more than $15 \%$ in LVESVI after 12 months of biventricular pacing, while $35 \%$ of the patients had increased LVESVI, and 14\% of the patients had increases of more than $15 \%$ in LVESVI. Sairaku, et $a{ }^{20)}$ compared the CRT efficacies in patients with LVEF $\leq 35 \%$ and QRS duration $\geq 120 \mathrm{~ms}$, patients with treatment upgraded to CRT, and AVB patients with LVEF $\leq$ $50 \%$ and found significantly reduced LVESV in the first 2 groups $(P=0.001)$ and no remarkable decrease in the last group $(P=0.9)$. In this study, only $47.2 \%$ of the patients showed responses to CRT. The results from the above studies suggest that variation in CRT efficacy is present in AVB patients with HF; therefore, it is necessary to identify preoperative predictive indicators for efficacy to facilitate the screening of patients. However, no definitive screening parameters are currently available. The results of this study show that QRS duration is an independent predictor of CRT efficacy in patients with third-degree AVB accompanied by HF. The patients with QRS duration $\geq 150 \mathrm{~ms}$ exhibited a superior response rate, myocardial remodeling reversal, and prognosis compared with the patients with QRS duration $<150 \mathrm{~ms}$. Although the baseline QRS duration is closely related to the escape site in patients with third-degree AVB, patients with a narrow QRS duration also showed no significant abnormalities in cardiac conduction or significant dyssynchrony. Considering the results of this study, direct implantation of CRT is not recommended for these patients. This study suggests that baseline QRS duration can be used as a predictor of CRT efficacy in patients with third-grade AVB accompanied by HF.

The QRS duration in patients with $\mathrm{HF}$ is associated with LVEF and NYHA classification and gradually widens with disease progression. ${ }^{21,22)}$ Patients with baseline QRS duration < $130 \mathrm{~ms}$ exhibited increased QRS duration after biventricular pacing, with poor response to CRT and even a possibly increased risk of death. ${ }^{23,24)}$ However, patients with baseline QRS duration $\geq 150 \mathrm{~ms}$ showed narrowed QRS duration following biventricular pacing, with significantly improved cardiac functions. ${ }^{25-27)}$ In patients with third-degree AVB, pacing QRS duration was not associated with the location of the conduction block ${ }^{28)}$ but was correlated with baseline QRS duration. ${ }^{28,29)}$ In this study, patients with QRS duration $<120 \mathrm{~ms}$ showed significantly widened QRS duration after biventricular pacing, with poor response to CRT, while patients with QRS duration $\geq 150 \mathrm{~ms}$ had remarkably shortened QRS duration following biventricular pacing, with a response rate to CRT as high as $76.5 \%$. The results of this study are consistent with the findings from previous studies. Meanwhile, we also found that a small number of patients with postoperative QRS widening in the group of QRS duration $<120 \mathrm{~ms}$ still responded to CRT. Similar observations were reported in the BLOCK HF study, some patients in the right ventricular pacing group also exhibited significant left ventricular remodeling, which may be associated with improved bradycardia by pacing instead of biventricular pacing or right ventricular pacing itself. Meta analyses,${ }^{30)}$ including RESPOND, RethinQ and LESSEREARTH, showed that the reductions in LVEDV and LVESV after CRT in patients with narrow QRS duration were more significant than those in the group treated with optimized drug therapy (or implantable cardioverterdefibrillator). The cause of the above results is still unclear, but the results suggested the presence of other factors that affect CRT efficacy, in addition to QRS duration. In the BLBS-HF study, which was aimed at patients with AVB accompanied by heart failure, $52 \%$ of the patients in the right ventricular pacing group showed declines in LVESVI $^{7)}$; AVB itself may be one of the causes of the above phenomenon. Although a small portion of the patients with narrow QRS durations exhibited improvements in myocardial remodeling after CRT, the overall efficacy was poor; therefore, CRT implantation is not recommended in this type of patient.

Limitations: The QRS duration of the patients with thirddegree AVB is affected by a variety of factors, such as es- 
cape site and ventricular conduction block, and its value in assessment of electrical dyssynchrony is less significant than that in patients with self-atrioventricular conduction. The assessment of its value would be more accurate with preoperative electrophysiological examination. The finding that the prognosis of the patients in group A was worse than that in the other 2 groups was possibly related to $\mathrm{HF}$ episodes as the main component of end point events as well as underlying diseases in addition to the differences in cardiac remodeling before and after CRT among the 3 groups. This study was a single-center study, with a small sample size and a short follow-up period. However, the impact of pacing on cardiac functions may take a longer amount of time; thus, studies with larger sample sizes and longer follow-up periods are needed to confirm the conclusions from this study.

\section{Conclusions}

This study confirmed the efficacy of CRT in patients with third-degree AVB accompanied by HF. To further improve the responses of patients to CRT, CRT efficacy can be predicted preoperatively based on baseline QRS duration. A QRS duration of $\geq 150 \mathrm{~ms}$ is an effective predictive indicator for postoperative efficacy.

\section{Disclosures}

Conflicts of interest: The authors have no conflicts of interest to declare.

\section{References}

1. Ahmed FZ, Khattar RS, Zaidi AM, Neyses L, Oceandy D, Mamas M. Pacing-induced cardiomyopathy: pathophysiological insights through matrix metalloproteinases. Heart Fail Rev 2014; 19: 669-80.

2. Kiehl EL, Makki T, Kumar R, et al. Incidence and predictors of right ventricular pacing-induced cardiomyopathy in patients with complete atrioventricular block and preserved left ventricular systolic function. Heart Rhythm 2016; 13: 2272-8.

3. Da Costa A, Gabriel L, Romeyer-Bouchard C, et al. Focus on right ventricular outflow tract septal pacing. Arch Cardiovasc Dis 2013; 106: 394-403.

4. Flevari P, Leftheriotis D, Fountoulaki K, et al. Long-term nonoutflow septal versus apical right ventricular pacing: relation to left ventricular dyssynchrony. Pacing Clin Electrophysiol 2009; 32: 354-62.

5. Martinelli Filho M, de Siqueira SF, Costa R, et al. Conventional versus biventricular pacing in heart failure and bradyarrhythmia: the COMBAT study. J Card Fail 2010; 16: 293-300.

6. Curtis AB, Worley SJ, Adamson PB, et al. Biventricular versus Right Ventricular Pacing in Heart Failure Patients with Atrioventricular Block (BLOCK HF) Trial Investigators. Biventricular pacing for atrioventricular block and systolic dysfunction. $\mathrm{N}$ Engl J Med 2013; 368: 1585-93.

7. St John Sutton M, Plappert T, Adamson PB, et al. Left ventricular reverse remodeling with biventricular versus right ventricular pacing in patients with atrioventricular block and heart failure in the BLOCK HF Trial. Circ Heart Fail 2015; 8: 510-8.

8. Ponikowski P, Voors AA, Anker SD, et al. 2016 ESC Guidelines for the diagnosis and treatment of acute and chronic heart failure: The Task Force for the diagnosis and treatment of acute and chronic heart failure of the European Society of Cardiology
(ESC)Developed with the special contribution of the Heart Failure Association (HFA) of the ESC. Eur Heart J 2016; 37: 2129200.

9. Hsing JM, Selzman KA, Leclercq C, et al. Paced left ventricular QRS width and ECG parameters predict outcomes after cardiac resynchronization therapy: PROSPECT-ECG substudy. Circ Arrhythm Electrophysiol 2011; 4: 851-7.

10. Ypenburg C, van Bommel RJ, Borleffs CJ, et al. Long-term prognosis after cardiac resynchronization therapy is related to the extent of left ventricular reverse remodeling at midterm follow-up. J Am Coll Cardiol 2009; 53: 483-90.

11. Khurshid S, Epstein AE, Verdino RJ, et al. Incidence and predictors of right ventricular pacing-induced cardiomyopathy. Heart Rhythm 2014; 11: 1619-25.

12. Ooka J, Tanaka H, Hatani Y, et al. Risk stratification of future left ventricular dysfunction for patients with indications for right ventricular pacing due to bradycardia. Int Heart J 2017; 58: 724-30.

13. Khurshid S, Liang JJ, Owens A, et al. Longer paced QRS duration is associated with increased prevalence of right ventricular pacing-induced cardiomyopathy. J Cardiovasc Electrophysiol 2016; 27: 1174-9.

14. Sakatani T, Sakamoto A, Kawamura K, et al. Clinical outcome after permanent pacemaker implantation in patients with a high percentage of ventricular pacing. Int Heart J 2015; 56: 622-5.

15. Molina L, Sutton R, Gandoy W, et al. Medium-term effects of septal and apical pacing in pacemaker-dependent patients: a double-blind prospective randomized study. Pacing Clin Electrophysiol 2014; 37: 207-14.

16. Singh H, Patel CD, Sharma G, Naik N. Comparison of left ventricular systolic function and mechanical dyssynchrony using equilibrium radionuclide angiography in patients with right ventricular outflow tract versus right ventricular apical pacing: A prospective single-center study. J Nucl Cardiol 2015; 22: 90311.

17. Kaye GC, Linker NJ, Marwick TH, et al. Effect of right ventricular pacing lead site on left ventricular function in patients with high-grade atrioventricular block: results of the ProtectPace study. Eur Heart J 2015; 36: 856-62.

18. Kindermann M, Hennen B, Jung J, Geisel J, Böhm M, Fröhlig G. Biventricular versus conventional right ventricular stimulation for patients with standard pacing indication and left ventricular dysfunction: the Homburg Biventricular Pacing Evaluation (HOBIPACE). J Am Coll Cardiol 2006; 47: 1927-37.

19. Curtis AB, Worley SJ, Chung ES, Li P, Christman SA, Sutton MS. Improvement in clinical outcomes with biventricular versus right ventricular pacing: the BLOCK HF Study. J Am Coll Cardiol 2016; 67: 2148-57.

20. Sairaku A, Yoshida Y, Nakano Y, et al. Don't expect left ventricular reverse remodeling after cardiac resynchronization therapy in patients with systolic heart failure and atrioventricular block: a multicenter study. Int J Cardiol 2016; 221: 597-600.

21. Iuliano S, Fisher SG, Karasik PE, Fletcher RD, Singh SN, Department of Veterans Affairs Survival Trial of Antiarrhythmic Therapy in Congestive Heart Failure. QRS duration and mortality in patients with congestive heart failure. Am Heart J 2002; 143: 1085-91

22. Sandhu R, Bahler RC. Prevalence of QRS prolongation in a community hospital cohort of patients with heart failure and its relation to left ventricular systolic dysfunction. Am J Cardiol 2004; 93: 244-6.

23. Thibault B, Harel F, Ducharme A, et al. Cardiac resynchronization therapy in patients with heart failure and a QRS complex < 120 milliseconds: the Evaluation of Resynchronization Therapy for Heart Failure (LESSER-EARTH) trial. Circulation 2013; 127: 873-81.

24. Ruschitzka F, Abraham WT, Singh JP, et al. Cardiacresynchronization therapy in heart failure with a narrow QRS complex. N Engl J Med 2013; 369: 1395-405.

25. Bryant AR, Wilton SB, Lai MP, Exner DV. Association between 
QRS duration and outcome with cardiac resynchronization therapy: a systematic review and meta-analysis. J Electrocardiol 2013; 46: 147-55

26. Chen JY, Lin KH, Chang KC, Chou CY. The shortest QRS duration of an electrocardiogram might be an optimal electrocardiographic predictor for response to cardiac resynchronization therapy. Int Heart J 2017; 58: 530-5.

27. Korantzopoulos P, Zhang Z, Li G, Fragakis N, Liu T. Metaanalysis of the usefulness of change in QRS width to predict response to cardiac resynchronization therapy. Am J Cardio 2016; 118: 1368-73.

28. Miyoshi F, Kobayashi Y, Itou H, et al. Prolonged paced QRS duration as a predictor for congestive heart failure in patients with right ventricular apical pacing. Pacing Clin Electrophysiol 2005; 28: 1182-8.

29. Pap R, Fürge P, Bencsik G, Makai A, Sághy L, Forster T. Native QRS complex duration predicts paced QRS width in patients with normal left ventricular function and right ventricular pacing for atrioventricular block. J Electrocardiol 2007; 40: 360-4.

30. Wang G, Zhao Z, Zhao S, Ding S, Shen S, Wang L. Effect of cardiac resynchronization therapy on patients with heart failure and narrow QRS complexes: a meta-analysis of five randomized controlled trials. J Interv Card Electrophysiol 2015; 44: 71-9. 